\title{
Systematic analysis of equatorial noise below the lower hybrid frequency
}

\author{
O. Santolík ${ }^{1}$, F. Němec ${ }^{1}$, K. Gereová ${ }^{1}$, E. Macúšová ${ }^{1}$, Y. de Conchy ${ }^{2}$, and N. Cornilleau-Wehrlin ${ }^{3}$ \\ ${ }^{1}$ Faculty of Mathematics and Physics, Charles University, V Holešovičkách 2, Prague, CZ-18000, Czech Republic \\ ${ }^{2}$ LESIA/Observatoire de Paris-Meudon, F-92195, Meudon Cedex, France \\ ${ }^{3}$ CETP/IPSL, 10/12 Avenue de L’Europe, Vélizy, F-78140, France
}

Received: 5 August 2003 - Revised: 24 November 2003 - Accepted: 11 December 2003 - Published: 14 July 2004

Part of Special Issue "Spatio-temporal analysis and multipoint measurements in space"

\begin{abstract}
We report results of a systematic analysis of a large number of observations of equatorial noise between the local proton cyclotron frequency and the local lower hybrid frequency. The analysis is based on the data collected by the STAFF-SA instruments on board the four Cluster spacecraft. The data set covers their first two years of measurement in the equatorial magnetosphere at radial distances between 3.9 and 5 Earth radii. Inspection of 781 perigee passages shows that the occurrence rate of equatorial noise is approximately $60 \%$. We identify equatorial noise by selecting data with nearly linearly polarized magnetic field fluctuations. These waves are found within $10^{\circ}$ of the geomagnetic equator, consistent with the published past observations. Our results show that equatorial noise has the most intense magnetic field fluctuations among all the natural emissions in the given interval of frequencies and latitudes. Electric field fluctuations of equatorial noise are also more intense compared to the average of all detected waves. Equatorial noise thus can play a non-negligible role in the dynamics of the internal magnetosphere.
\end{abstract}

Key words. Magnetospheric physics (waves in plasma) Space plasma physics (waves and instabilities) - Radio science (magnetospheric physics)

\section{Introduction}

Equatorial noise is an intense natural emission of electromagnetic plasma waves observed within a few Earth radii $\left(R_{E}\right)$ of geocentric radial distance, and always recorded very close to the geomagnetic equator. Its frequency interval ranges from a few Hertz to several hundreds of Hertz. These waves were first observed by Russell et al. (1970) in the outer plasmasphere at frequencies between twice the local proton cyclotron frequency $\left(f_{\mathrm{H}^{+}}\right)$and half the lower hybrid frequency $\left(f_{\mathrm{lh}}\right)$.

Correspondence to: O. Santolík

(ondrej.santolik@mff.cuni.cz)
The observations were made within $2^{\circ}$ from the equator, and the magnetic field fluctuations carried by those waves were found to be very close to the direction parallel to the static terrestrial magnetic field $(\boldsymbol{B})$. This observed polarization corresponds well to the theoretical properties of the whistler-mode waves below $f_{\text {lh }}$ (e.g. Stix, 1992), assuming that the wave vectors are very close to perpendicular to $\boldsymbol{B}$.

Later observations (Gurnett, 1976; Perraut et al., 1982; Laakso et al., 1990; Kasahara et al., 1994; André et al., 2002) revealed that the equatorial noise occurs at radial distances between 2 and $7 \mathrm{R}_{\mathrm{E}}$, and at latitudes within $10^{\circ}$ from the magnetic equator, and that its lowest frequency could go down to the fundamental $f_{\mathrm{H}^{+}}$. Detailed time-frequency spectrograms (Gurnett, 1976) also showed that, what appears as a noise band in the low resolution data, is in fact a superposition of many spectral lines with different frequency spacings. The term "equatorial noise" is, however, still used even for these discrete wave phenomena, to indicate the connection to the original observations of Russell et al. (1970). The generation mechanism of these waves is most probably connected to the ion-cyclotron harmonic interaction (Gurnett, 1976), with energetic protons having ring-like distribution functions at a pitch angle of $90^{\circ}$ (Perraut et al., 1982). After being generated at the ion Bernstein wave-mode branches, the waves subsequently propagate in the electromagnetic whistler mode (also known as the extraordinary or fast magnetosonic mode). Equatorial noise thus can be observed far away from its generation region.

Multipoint observations of equatorial noise by the four Cluster spacecraft have been presented by CornilleauWehrlin et al. (2003) and Santolík et al. (2002). They addressed the location of noise with respect to the equatorial plane and the spatio-temporal structure of its frequency spectra. Santolík et al. (2002) found these waves within $2^{\circ}$ of the magnetic equator, and Cornilleau-Wehrlin et al. (2003), in another case, at about $3^{\circ}$ of the model equator, with an extension of $0.15 \mathrm{R}_{\mathrm{E}}$ in the direction perpendicular to the ecliptics. Since a common feature of the equatorial noise is the presence of harmonic lines whose spacings do not match the local 
(a)
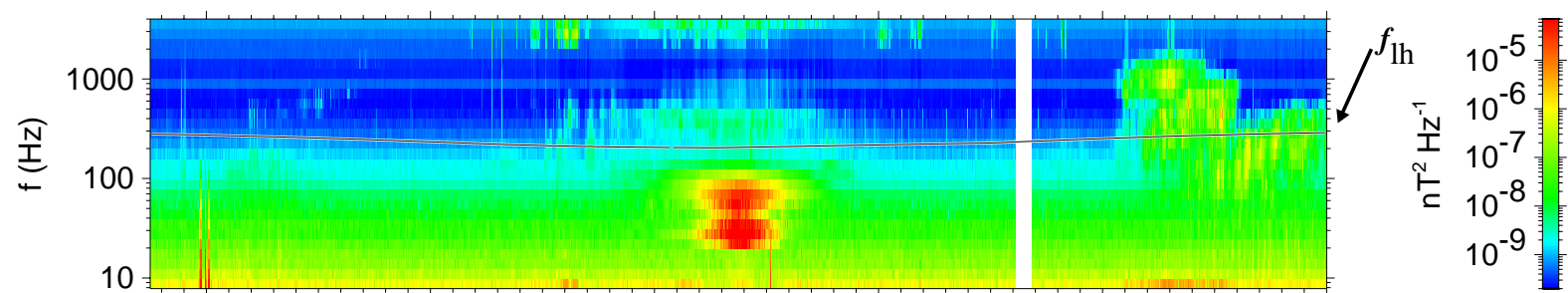

(b)

(c)
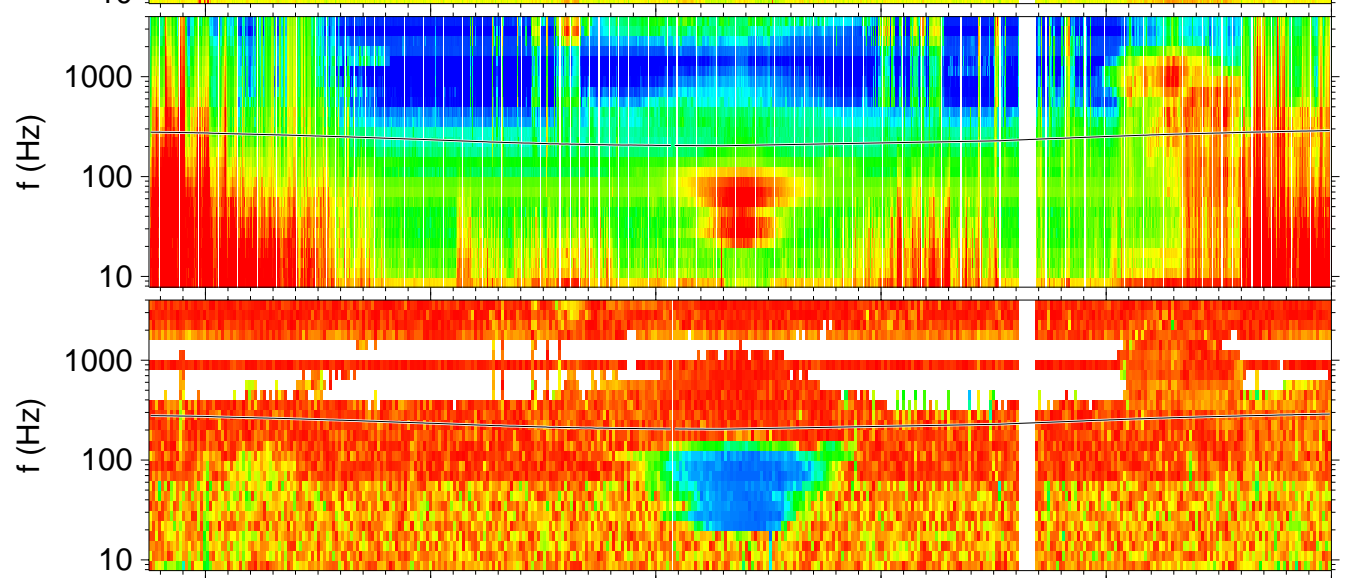

(d)

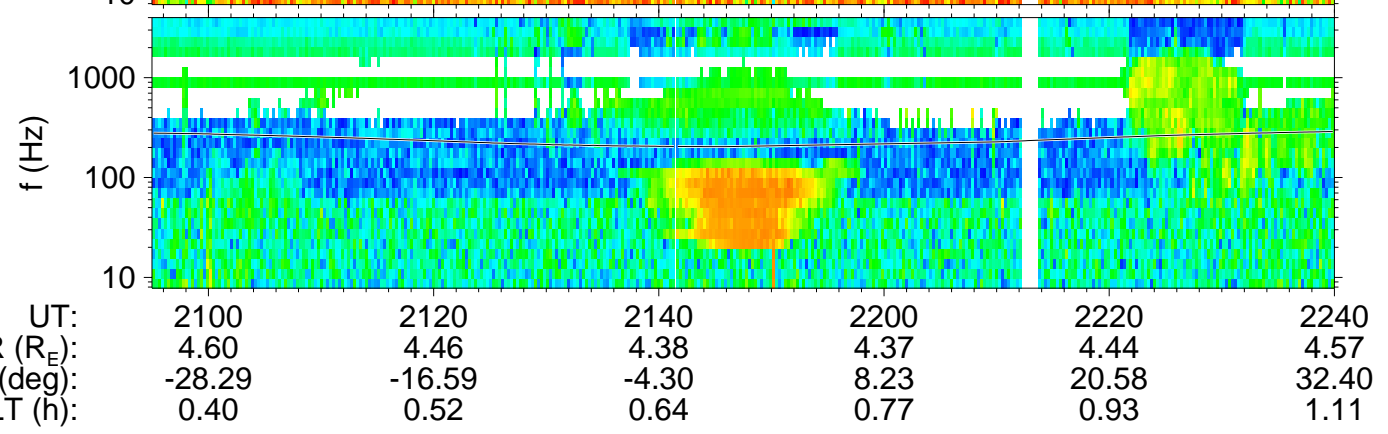

Fig. 1. Example of data collected by Cluster 4 on 17 February 2002. From the top: (a) sum of the power-spectral densities of the three magnetic components, (b) sum of the power-spectral densities of the two electric components ; (c) ellipticity and (d) planarity are determined using the singular value decomposition of the magnetic spectral matrix. Universal time (UT) and position of the spacecraft are given on the bottom of the figure using the radial distance $(\mathrm{R})$ in the Earth radii $\left(\mathrm{R}_{\mathrm{E}}\right)$, magnetic dipole latitude (MLat) in degrees, and magnetic local time (MLT) in hours. Maximum possible value of the local lower hybrid frequency $\left(f_{\mathrm{lh}}\right)$ is plotted over the panels (a)-(d). The data in panels (c) and (d) are not shown for weak signals below $10^{-9} \mathrm{nT}^{2} \mathrm{~Hz}^{-1}$.

$f_{\mathrm{H}^{+}}$, waves have to propagate in the radial direction, in order to move through the equatorial plane to a place with a different magnetic field strength. Santolík et al. (2002) showed a case where the wave propagation directions have a radial component.

This paper is also based on the data of the Cluster project. We present the first results of our systematic analysis of a large number of observations of equatorial noise. These emissions are, together with the whistler mode chorus, among the most intense electromagnetic waves observed in the low-latitude region of the Earth's magnetosphere, and their sensitivity to the variations of the geomagnetic activity indicates their importance for the "space weather" applications, i.e. prediction of fluxes of energetic particles as a consequence of variations of the solar input (André et al., 2002). Therefore, we focus our attention on the probability densities of the wave intensity in both electric and magnetic com- ponents. We establish a selection criterion for recognizing the equatorial noise emissions, and compare the intensities of equatorial noise with those of other emissions in the same interval of frequencies and latitudes. In Sect. 2 we will present the data set and, using an example case, we will describe our analysis methods. In Sect. 3 we will show results of the systematic analysis, in Sect. 4 we will discuss these results and, finally, in Sect. 5 we will present brief conclusions.

\section{Data set and analysis methods}

We use the data collected by the "spatiotemporal analysis of field fluctuations" (STAFF-SA) instruments on board the four Cluster spacecraft operated by the European Space Agency (ESA). STAFF-SA was designed for onboard calculation of power-spectral densities, mutual phases, and coherence relations of three orthogonal magnetic components and 
two electric components (Cornilleau-Wehrlin et al., 1997, 2003). The analysis is made in 27 frequency channels between $8 \mathrm{~Hz}$ and $4 \mathrm{kHz}$, and the instrument has several measurement modes. Of those, we only use the "normal mode" in the present study. This mode provides us with the full five-component analysis in the entire frequency band of the instrument. The data are analyzed on board with the time resolution of $1 \mathrm{~s}$ (for the power-spectral densities) or $4 \mathrm{~s}$ (for the phases and coherence). The results are then compressed and transmitted to the ESA receiving stations. In the subsequent preprocessing phase the received data are calibrated and organized in Hermitian spectral matrices $5 \times 5$, one matrix per frequency channel, i.e. 27 matrices every 4 s. Each spectral matrix contains the real power-spectral densities on its main diagonal and complex cross-spectral densities as its off-diagonal elements.

We have analyzed all the preprocessed data intervals where the Cluster spacecraft were close to their perigee during the first two years of operation (2001-2002). In these portions of their orbits the spacecraft scanned the lowlatitude region of the Earth's magnetosphere at all magnetic local times, and at radial distances between 3.9 and $5 \mathrm{R}_{\mathrm{E}}$. The preprocessed STAFF-SA data have been organized into intervals of $\approx 1$-hour duration, and we have selected those intervals where the instrument measured within $\approx 30^{\circ}$ of the geomagnetic equator. In most cases we have joint two or three adjacent $\approx 1$-hour intervals, to obtain a better coverage of the equatorial region. We have worked with the data of each spacecraft separately, thus increasing the amount of data intervals entering in the statistics. Discussion of this approach is presented in Sect. 4. Using that procedure, we have collected the total number of 781 data intervals of $\approx 1-3 \mathrm{~h}$, measured in the equatorial region close to the perigee of the four Cluster spacecraft. To ensure the coherence of our results and to gain experience for their reasonable interpretation, we have visually inspected all the intervals before doing the subsequent computer analysis. This inspection showed that in 671 perigee cases the data were available in a restricted interval of latitudes within $10^{\circ}$ of the geomagnetic equator where we expect equatorial noise to appear. Of those cases, we have identified the presence of equatorial noise in 398 intervals (59\%).

Figure 1 shows an example of those data intervals where the equatorial noise emissions were present. The measurements were done by Cluster 4 on 17 February 2002. The spacecraft was close to its perigee, and we show the data recorded in the low-latitude region within $\pm 30^{\circ}$ of magnetic equator (magnetic latitude is determined using the dipole approximation of the Earth's magnetic field). Figures $1 \mathrm{a}$ and $1 \mathrm{~b}$ represent power-spectral densities of the magnetic and electric field, respectively. Equatorial noise is the intense electromagnetic emission seen on both panels close to the center of the time interval, within a few degrees from the equator. In the frequency domain it appears as two main peaks at $\approx 30 \mathrm{~Hz}$ and $\approx 70 \mathrm{~Hz}$. The emission is confined below the upper estimate of the lower hybrid frequency $\left(f_{\text {ih }}\right)$, calculated as the geometric average of the proton and electron cyclotron fre- quencies. To plot this estimate in Fig. 1, we use the measurements of the ambient magnetic field made on board (Balogh et al., 2001). It represents the true value of $f_{\text {lh }}$ only in a dense plasma, with the plasma frequency much larger than the electron cyclotron frequency, which is always the case in the plasmasphere.

Multidimensional measurements of electric and magnetic fields allow us to analyze polarization properties of equatorial noise emissions. Ellipticity $L_{p}$ of polarization of the magnetic field fluctuations is shown in Fig. 1c. We use the singular value decomposition technique from Eq. (13) of Santolík et al. (2003), defining $L_{p}=w_{2} / w_{1}$, where $w_{1}$ and $w_{2}$ are the two largest singular values of the magnetic spectral matrix. In the idealized case of exactly planar polarization, the result represents the ratio of the lengths of the minor and major axes of the polarization ellipse. It varies between 0 (linear polarization) and 1 (circular polarization). The equatorial noise can be easily distinguished by its polarization close to linear, as it was first described by Russell et al. (1970). Very similar results have been obtained using classical methods based on eigenvalue analysis (e.g. Samson, 1973). The abrupt change in the $L_{p}$ values at a constant frequency of $62.5 \mathrm{~Hz}$ outside the equatorial noise emission is an instrumental effect. It is connected to the boundary between the frequency bands of the STAFF-SA instrument, where different averaging of measured data is done during the onboard analysis.

Figure $1 \mathrm{~d}$ represents the planarity $F$ of the polarization of magnetic field fluctuations from Eq. (12) of Santolík et al. (2003). It is defined as $F=1-\sqrt{ } w_{3} / \sqrt{ } w_{1}$, where $w_{1}$ and $w_{3}$ are, respectively, the maximum and the minimum singular values of the magnetic spectral matrix. In an idealized case of random fluctuations, $F$ reflects the ratio of the shortest and the longest axes of the 3-D polarization ellipsoid, defined by standard deviations of magnetic noise. A value close to 0 would mean that the fluctuations appear in all three axes of the ellipsoid with the same probability, whereas a value of 1 would represent a strict confinement of the fluctuations to a single 2-D plane. Note that the boundary at $62.5 \mathrm{~Hz}$ seen in Fig. 1d is the same instrumental effect as described in the previous paragraph. For the equatorial noise, the value of $F \approx 0.8$ suggests that the magnetic field fluctuates very close to a single plane, with a small fraction of random 3-D fluctuations. Values of $F$ and $L_{p}$ are obviously interdependent in that sense, that a given $L_{p}$ sets a lower limit of possible values of $F, 1-\sqrt{L_{p}} \leq F \leq 1$. On the other hand, a given $F$ doesn't have any implication for possible values of $L_{p}$.

\section{Systematic analysis of the entire data set}

The polarization analysis shown in Fig. 1 has been done with the entire data set recorded during the perigee portions of the 781 orbits of the Cluster spacecraft. We have selected the frequency channels between $8 \mathrm{~Hz}$ (the lowest frequency analyzed by the STAFF-SA instrument) and $300 \mathrm{~Hz}$ (the upper estimate of the maximum $f_{\mathrm{lh}}$ throughout the data set). This 
(a)

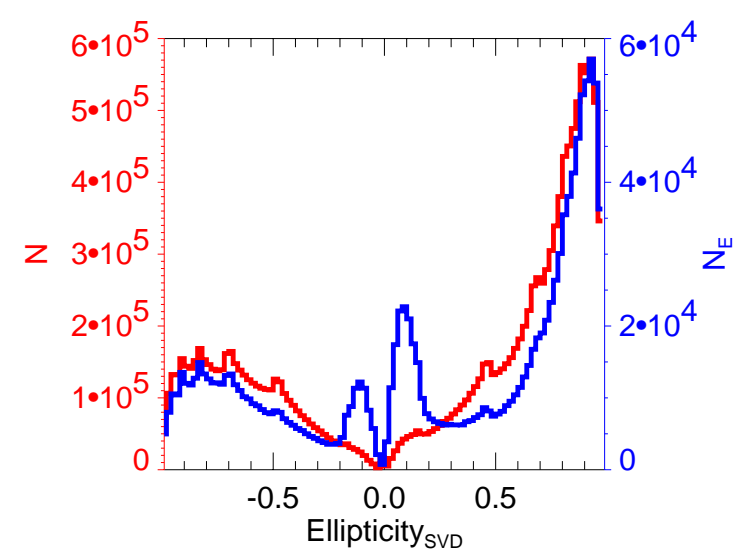

(b)

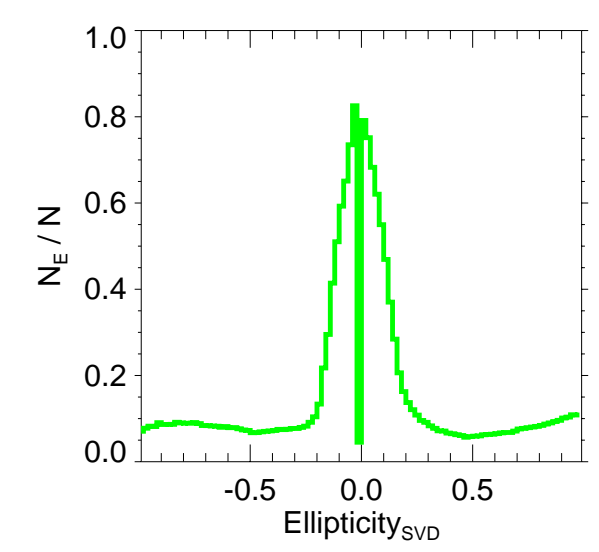

Fig. 2. Histograms of modified ellipticity $L_{S} L_{p}$ (see text) determined using the singular value decomposition of the magnetic spectral matrix. (a) Histogram of all $1.4 \times 10^{7}$ time-frequency points, plotted in red with the left-hand-side vertical scale; and of selected $1.3 \times 10^{6}$ intense events above $10^{-6} \mathrm{nT}^{2} \mathrm{~Hz}^{-1}$, plotted in blue, with the right-hand-side vertical scale. (b) Normalized histogram of the elipticity of the intense events.

means that we use only the 16 lowest frequency channels of the instrument. The analysis has been further limited to the data measured within $\pm 30^{\circ}$ of magnetic latitude from the magnetic equator. If this interval of latitudes is completely covered by the data, it would, given the orbital parameters of Cluster satellites, correspond to a time interval of approximately $1 \mathrm{~h}$ and $40 \mathrm{~min}$, i.e. with the 4 -second time resolution, to 1500 measurements. Since the selected time intervals do not always cover that total range of latitudes, the average number in our data set is $\approx 1100$ measurements per interval. For the 781 perigee passages, we thus have the total number of $16 \times 1100 \times 781 \approx 1.4 \times 10^{7}$ time-frequency points. In these points we have analyzed measured spectral matrices which served as input data for calculations of the average power-spectral densities of the magnetic and electric fluctuations, ellipticity, and planarity (see Sect. 2).

Figure 2 shows histograms constructed from the obtained ellipticity values. The results are now slightly modified compared to the method used in Fig. 1c. The purpose is to reflect the sense of rotation of the wave magnetic field. We (a)

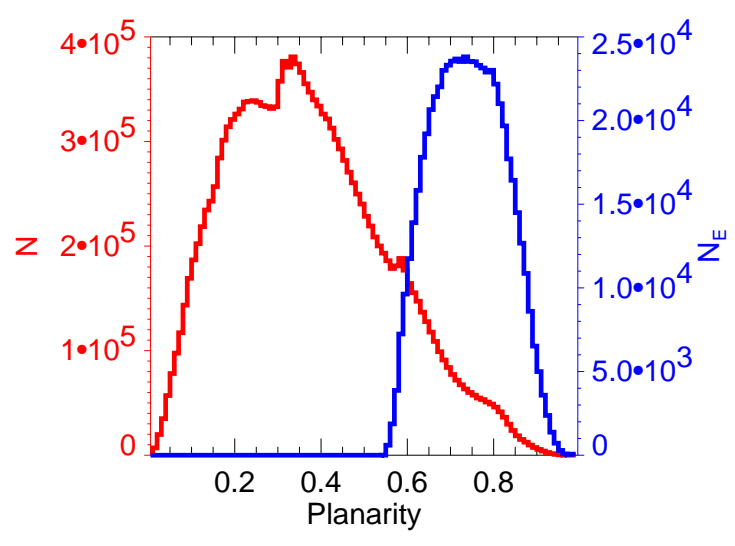

(b)

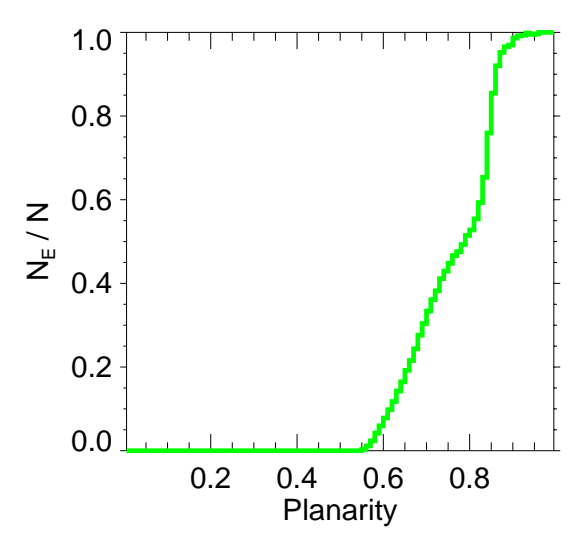

Fig. 3. Histograms of the planarity determined using the singular value decomposition of the magnetic spectral matrix. (a) Histogram for all $1.4 \times 10^{7}$ time-frequency points, plotted in red with the lefthand-side vertical scale; and for selected $5.4 \times 10^{5}$ nearly linearly polarized events with $L_{p}<0.2$, plotted in blue, with the righthand-side vertical scale. (b) A normalized histogram for the nearly linearly polarized waves.

multiply $L_{p}$ by a sign coefficient $L_{s}$, which is either -1 , or +1 according to the sign of the phase shift between the two components of magnetic fluctuations perpendicular to $\boldsymbol{B}$. The value of -1 represents the left-hand polarized waves (the sense of the ion cyclotron motion) and the value of +1 represents the right-hand polarized waves, (the sense of the electron cyclotron motion). If the polarization is linear, and the corresponding phase shifts are either $0^{\circ}$ or $\pm 180^{\circ}$, then $L_{p}$ is zero and $L_{s}$ may be defined as -1 or +1 , with no effect on the resulting product $L_{s} L_{p}$. Similarly, if the polarization is close to linear, $L_{p}$ is close to zero, and the influence of $L_{s}$ on the result is negligible. The values $L_{s} L_{p}$ of \pm 1 then correspond to exactly circular polarization (right- or left-handed), absolute values less than 1 mean elliptic polarization, and the value of zero still represents strictly linearly polarized waves.

To obtain the histograms in Fig. 2 , the interval $\langle-1,+1\rangle$ has been divided into 100 consecutive subintervals, and the number of cases contained in each of these subintervals has been counted. The entire data set mainly contains right-hand, nearly circularly polarized waves with some small fraction of 


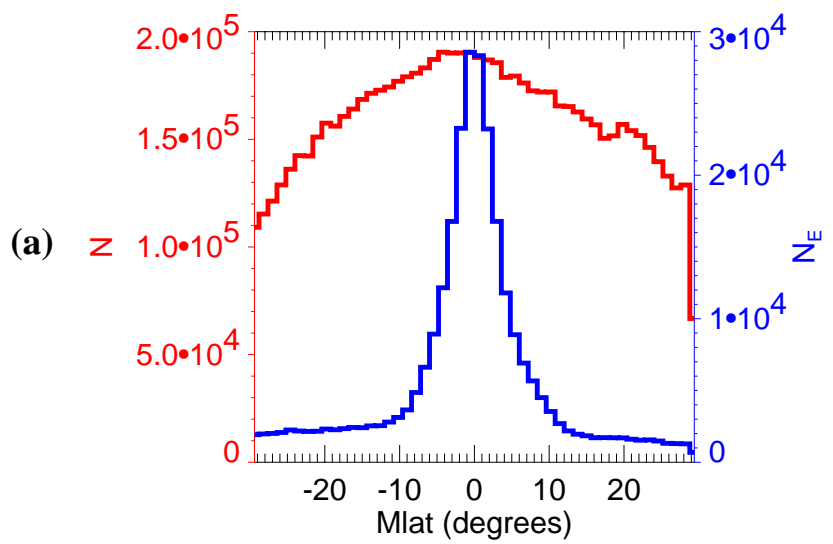

(a)

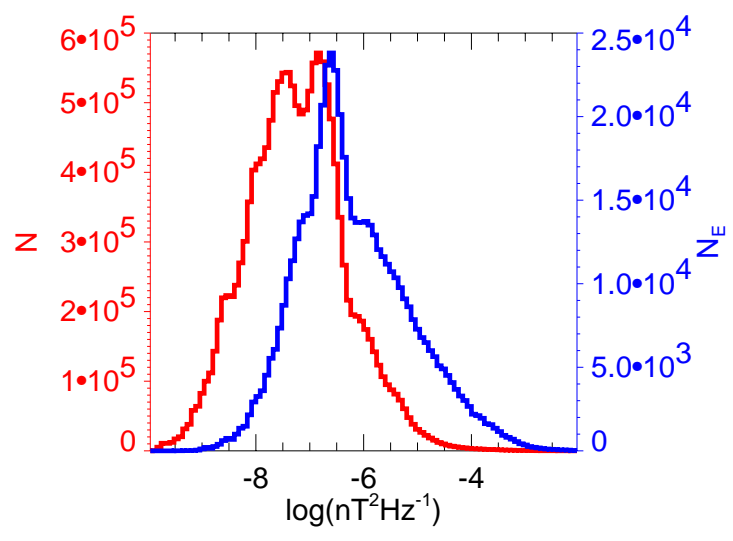

(b)
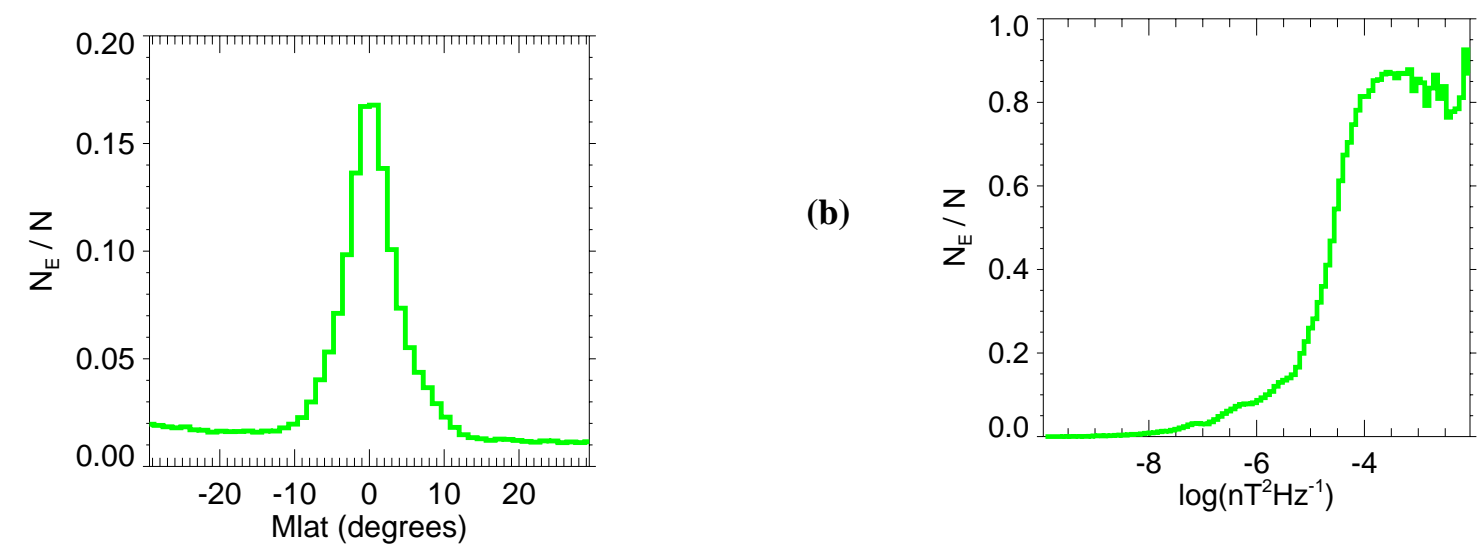

Fig. 4. The same as in Fig. 3, but for the magnetic dipole latitude.

left-hand polarized waves (red curve in Fig. 2a). Note that the onboard compression algorithm leads to phase quantization effects reflected on the histogram as a set of superposed small-amplitude peaks. If we now select just the most intense waves with the magnetic spectral densities larger than $10^{-6} \mathrm{nT}^{2} \mathrm{~Hz}^{-1}$, we obtain a non-negligible fraction of nearly linearly polarized waves with the absolute value of ellipticity below $\approx 0.2$ (blue curve in Fig. $2 a$ ). The artifacts introduced by the onboard compression are removed if we calculate the relative fraction of the intense waves among all the recorded measurements. This normalized histogram of ellipticity (Fig. 2b) is obtained as the ratio of the blue and red histograms from Fig. 2a. It clearly shows that the majority of nearly linearly polarized waves is more intense than $10^{-6} \mathrm{nT}^{2} \mathrm{~Hz}^{-1}$, forming a distinct peak around zero ellipticity in Fig. 2b, with a maximum close to $80 \%$. Based on our visual inspection of all the available data from the perigee passages of the Cluster satellites, we can identify those intense linearly polarized waves with the equatorial noise emissions. As we will show next, this identification proves true, considering our results on the magnetic latitudes of these waves. From Fig. $2 b$ we can also estimate a reasonable threshold of 0.2 for the ellipticity $L_{p}$, bounding the peak of the intense linearly polarized waves. This threshold will be used in the following to identify the equatorial noise emissions.

(b)

Fig. 5. The same as in Fig. 3, but for the sum of the power-spectral densities of the three components of magnetic field fluctuations.

Results for the planarity of the magnetic field polarization are shown in Fig. 3. We use the same analysis method as for the example case in Fig. 1d. The obtained values have been counted in 100 subintervals between 0 and 1 to construct the histograms in Fig. 3. The histogram for all events (red curve in Fig. 3a) shows a broad peak shifted toward lower values, with a maximum around 0.3 . For the selected nearly linearly polarized waves with $L_{p}<0.2$ (blue curve in Fig. 3a) the peak is clearly moved towards higher values of the planarity. Its maximum is now slightly below 0.8 . As we have shown in Sect. 2, possible values of the planarity $F$ are limited, given the ellipticity $L_{p}$. For $L_{p}<0.2$ we cannot obtain $F$ lower than $\approx 0.55$. Normalized histogram of the planarity values for the nearly linearly polarized waves is shown in Fig. 3b. We can see that $100 \%$ of the observations of high planarity values $(>0.9)$ correspond to the nearly linearly polarized waves. This fraction decreases toward lower planarity values down to the cutoff at $F \approx 0.55$.

Figure 4 presents the histograms of the magnetic dipole latitudes of the events. To obtain these histograms, the interval from $-30^{\circ}$ to $+30^{\circ}$ has been divided into 50 consecutive subintervals. The histogram for all events (red curve and left-hand scale in Fig. 4a) is not a constant function of the magnetic latitude because of the particular method we used to select the data set. Since we always selected the data intervals containing the measurements close to the magnetic 
(a)

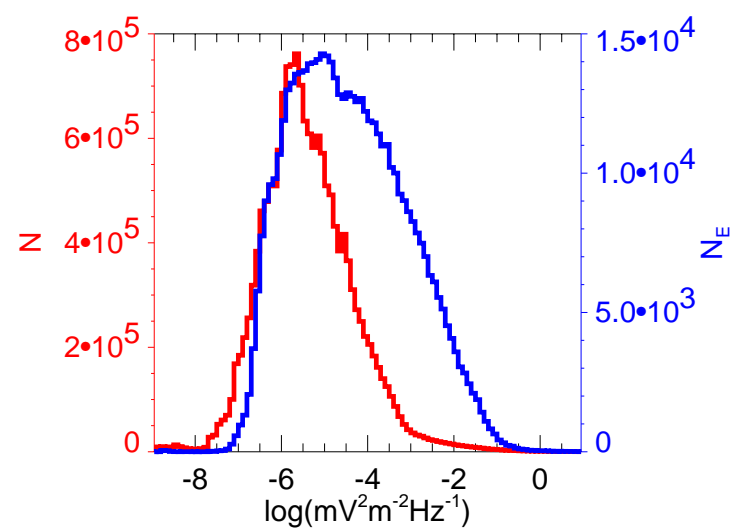

(b)

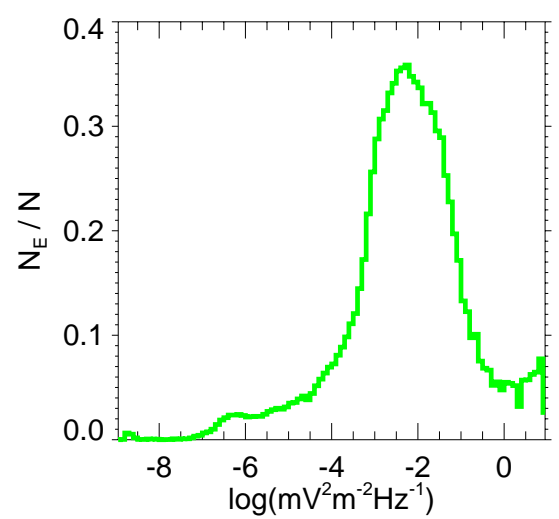

Fig. 6. The same as in Fig. 3, but for the sum of the power-spectral densities of the two measured components of electric field fluctuations.

equator, the histogram shows a broad peak centered at the zero latitude. This is therefore a purely artificial effect.

The histogram looks quite different for the waves with $L_{p}<0.2$ (blue curve and right-hand scale in Fig. 4a). The maximum is again close to the equator, but the peak is now much narrower, with the majority of events confined within $10^{\circ}$ of the magnetic equator. The ratio of the two histograms, shown in Fig. 4b, again forms a narrow peak, with approximately the same width. It moreover shows that about $17 \%$ of all events observed close to the magnetic equator is nearly linearly polarized. This fraction decreases toward higher latitudes. For latitudes to the south of $-10^{\circ}$ and to the north of $+10^{\circ}$, it goes down to $1-2 \%$. This residual value will be discussed in Sect. 4. The majority of the observed nearly linearly polarized waves is, however, clearly concentrated close to the equator. This further validates this selection criterion for the equatorial noise emissions.

In the analysis presented in Fig. 2 we have used a threshold of $10^{-6} \mathrm{nT}^{2} \mathrm{~Hz}^{-1}$ to select intense waves. Figure 5 shows more details on the distribution of the obtained values of the magnetic power-spectral density. To construct the histograms, the interval between $10^{-10}$ and $10^{-2} \mathrm{nT}^{2} \mathrm{~Hz}^{-1}$ has been divided into 100 consecutive logarithmic subintervals. The histogram of all events (red curve and left-hand scale in Fig. 5a) peaks close to $10^{-7} \mathrm{nT}^{2} \mathrm{~Hz}^{-1}$, steeply decreases just below $10^{-6} \mathrm{nT}^{2} \mathrm{~Hz}^{-1}$, and forms a decreasing tail at higher intensities.

The main peak is only slightly higher for the waves with $L_{p}<0.2$ (blue curve and right-hand scale in Fig. 5a), but the high-intensity tail extends to significantly higher intensities with a higher probability. The ratio of the two histograms, shown in Fig. 5b, reveals that the linearly polarized waves dominate the most intense emissions with the magnetic power-spectral density above $10^{-4} \mathrm{nT}^{2} \mathrm{~Hz}^{-1}$, leaving only about $20 \%$ of those intense events to waves with a different polarization. Oppositely, a very low relative fraction of nearly linearly polarized waves is observed for less intense waves below $10^{-6} \mathrm{nT}^{2} \mathrm{~Hz}^{-1}$.

Figure 6 shows histograms of the electric power-spectral density in a similar format as in Fig. 5. We use the interval of values between $10^{-9}$ and $10^{1} \mathrm{mV}^{2} \mathrm{~m}^{-2} \mathrm{~Hz}^{-1}$, again divided into 100 consecutive logarithmic subintervals. The histogram of all events (red curve in Fig. 6a) has a peak value close to $10^{-5} \mathrm{mV}^{2} \mathrm{~m}^{-2} \mathrm{~Hz}^{-1}$, about one order of magnitude lower than the histogram for the nearly linearly polarized waves (blue curve). Above $10^{-4} \mathrm{mV}^{2} \mathrm{~m}^{-2} \mathrm{~Hz}^{-1}$, the probability density for the $L_{p}<0.2$ subset of events is shifted by about two orders of magnitude higher. The relative fraction of the nearly linearly polarized waves (the ratio of the two histograms shown in Fig. 6b) doesn't reach such as high values as in the case of the magnetic field fluctuations. It shows a peak fraction of about $35 \%$ for relatively intense waves slightly below $10^{-2} \mathrm{mV}^{2} \mathrm{~m}^{-2} \mathrm{~Hz}^{-1}$, but for higher intensities the relative fraction again decreases down to about $5 \%$. This means that other types of polarization dominate at those extremely high intensities.

\section{Discussion}

The most important simplification in our analysis method is the selection criterion we use to recognize the equatorial noise emissions. To analyze large volumes of data it is inevitable to use an automatic recognition procedure, always taking the risk that some data points are either not selected when they should be, or selected by mistake. On the other hand, the advantage of this approach is that the selection is absolutely objective, based on a well-defined criterion, thus removing all possibilities for subjective case-by-case judgement which could bias the resulting statistics. In this study we have chosen to set up a relatively simple criterion based on the ellipticity of the magnetic field fluctuations, using known characteristics of the equatorial noise and the visual inspection of all the cases. To verify this approach we have checked the resulting statistics of magnetic latitude and planarity of polarization.

The only main problem of this selection criterion has been found in the analysis of magnetic latitudes (Fig. 4b). We have obtained a constant fraction of $1-2 \%$ of the nearly linearly polarized waves for magnetic latitudes below $-10^{\circ}$ and above $+10^{\circ}$. If we have no other explanation of these waves, it would mean that the linearly polarized equatorial noise 
extends in latitude at least $30^{\circ}$ from the magnetic equator. This would be in contradiction with the results published in the past. An alternative explanation could be that these observations correspond to linearly polarized signals that we wrongly classify as equatorial noise. We suppose the phenomenon is connected to the low-frequency magnetic noise of the instrument. We can see in Fig. 1c that the instrumental noise creates patchy appearances of nearly linear polarization well outside the compact time-frequency region of the natural equatorial noise. Since these spots of linear polarization are evenly distributed at all latitudes, the result is the observed 1-2\% floor on the normalized histogram.

To test this hypothesis we have further narrowed our selection criteria to include only the most intense waves, with the magnetic spectral density larger than $10^{-6} \mathrm{nT}^{2} \mathrm{~Hz}^{-1}$. This condition has been combined with the threshold $L_{p}<0.2$. In such a way, the influence of the weak instrumental noise has been eliminated. The resulting normalized histogram (Fig. 7) proves that the main peak close to the equator remains in place as we see it in Fig. 4b but the 1-2\% floor disappears. More precisely, the main peak reaches somewhat lower values of $\approx 10 \%$, and the fraction becomes negligible outside the interval of magnetic latitudes between $-10^{\circ}$ and $+10^{\circ}$. This confirms the hypothesis that the $1-2 \%$ floor in Fig. $4 \mathrm{~b}$ is owing to the instrumental noise.

In the present study, we have made another simplification in analyzing the data of the four Cluster spacecraft separately. The approach naturally increases the volume of data entering in the statistics by a factor of 4 . However, all the spacecraft pass through the equatorial region in relatively short intervals of time, from less than one minute up to about $45 \mathrm{~min}$, depending on the actual configuration of the spacecraft orbits. We can then question the hypothesis of the independence of the four data sets obtained by the different spacecraft. The geomagnetic conditions certainly are very similar for the four spacecraft during their closely separated perigee passages, but, nevertheless, the internal structure of the equatorial noise emissions can be highly variable at spatiotemporal scales comparable to the separation of the spacecraft (Santolík et al., 2002). In our data set we can find cases where the position of the peak intensity and the latitudinal extent of the equatorial noise are nearly the same on the four spacecraft, and other cases where these parameters are considerably different. Detailed analysis of these differences is beyond the scope of the present paper but for the purpose of this discussion we can conclude that the total number of independent cases can be up to 4 times lower, if we consider as independent only those passages separated by at least one full orbital period of the spacecraft ( 2 days and $9 \mathrm{~h}$ ). This still does not decrease the total volume of data below any reasonable limit of statistical reliability (recall that the total number of time-frequency points is $1.4 \times 10^{7}$ - after using the most severe selection criteria in Fig. 7 this number still reaches a value of $2 \times 10^{5}$ ).

The analysis of the electric and magnetic power-spectral densities is the main purpose of this work. The results show that, without any doubt, the nearly linearly polarized emis-

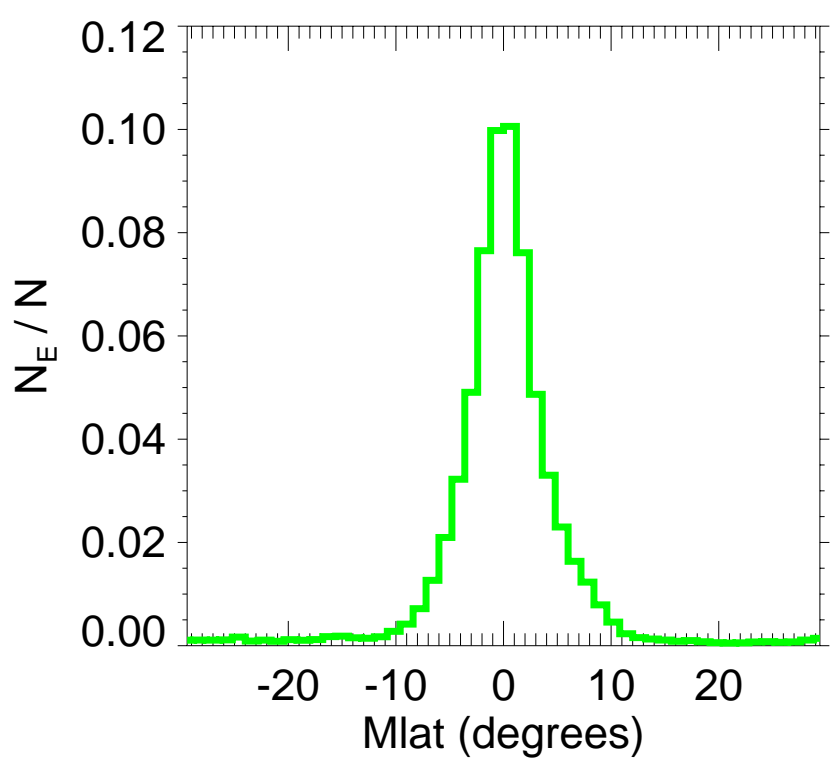

Fig. 7. The same as in Fig. 4b, but with the additional selection criterion of the magnetic spectral density larger than $10^{-6} \mathrm{nT}^{2} \mathrm{~Hz}^{-1}$.

sions are the most intense ones in the given interval of frequencies and latitudes, as concerns the magnetic field fluctuations. Electric field fluctuations of these emissions are also more intense compared to the average of all detected waves but still their relative fraction in Fig. 6b decreases at very high intensities of more than $10^{-2} \mathrm{mV}^{2} \mathrm{~m}^{-2} \mathrm{~Hz}^{-1}$. It then appears interesting to determine what kind of waves constitutes those in approximately $95 \%$ of the intense events. Here we must take into account that the selection criterion is based on the polarization of the magnetic fluctuations and does not take into account the polarization of the wave electric field. Intense electrostatic waves thus would not be selected even if they were linearly polarized because they are not accompanied by a magnetic field signal. Thus, we interpret these intense waves as broadband electrostatic noise, often occurring at higher latitudes which are magnetically connected to the Southern and Northern auroral regions. An example can be seen in Fig. 1b where broad-band electrostatic fluctuations are mainly observed at magnetic latitudes below $-25^{\circ}$ and above $+25^{\circ}$. Projected along the approximately dipolar magnetic field lines on the Earth's surface, these positions correspond, respectively, to auroral latitudes below $-65^{\circ}$, and above $+65^{\circ}$.

The final note of this discussion concerns the significance of our results. We have proven that equatorial noise is the most intense electromagnetic emission within $30^{\circ}$ of the geomagnetic equator, in the frequency range between $f_{\mathrm{H}^{+}}$and $f_{\mathrm{lh}}$, and in the range of radial distances between 4 and $5 \mathrm{R}_{\mathrm{E}}$. As we have shown in Sect. 2, equatorial noise is detected in approximately $60 \%$ of all passages of the Cluster spacecraft through the equatorial region at $4-5 \mathrm{R}_{\mathrm{E}}$. This high occurrence rate, together with the relatively high observed intensities, indicate that the importance of equatorial noise may be not as marginal, as would suggest the low number of papers 
concerning these emissions which can be found in the public literature. Our results suggest an analogy with natural emissions of whistler-mode chorus (generally observed above $f_{\mathrm{lh}}$ ), which are also very intense, and which are believed to significantly influence the dynamics of the energetic electrons in the Earth's outer radiation belt (Meredith et al., 2003; Horne et al., 2003; Horne and Thorne, 2003). According to the most accepted generation mechanism of equatorial noise (Gurnett, 1976; Perraut et al., 1982), its amplification is fed by the free energy in the distribution functions of energetic ions, which may be significantly influenced by interactions with these waves.

Natural emissions of equatorial noise can thus play a nonnegligible role in the dynamics of the internal magnetosphere. Equatorial noise can lead to modifications of the distribution functions of energetic ions, possibly causing their perpendicular heating or, on the other hand, phase-space diffusion and eventual precipitation into the Earth's atmosphere. All those arguments are, however, still rather speculative, and global effects of equatorial noise have yet to be quantitatively determined. Our results may indicate that this future research is worth being done.

\section{Conclusions}

We have described the first results of a systematic study of equatorial noise observed by the Cluster spacecraft between the local proton cyclotron frequency and the local lower hybrid frequency. This study has been based on the data measured by the STAFF-SA instrument during its first two years of operation. We have analyzed data collected during 781 perigee intervals of the four Cluster spacecraft at radial distances between 3.9 and 5Earth radii, and at magnetic latitudes between $-30^{\circ}$ and $+30^{\circ}$. Inspection of these intervals has shown that the occurrence rate of equatorial noise is approximately $60 \%$.

Polarization analysis of magnetic field fluctuations has allowed us to select nearly linearly polarized waves with ellipticities below 0.2. These waves have been found to have the highest planarity among all the collected wave observations. They have been mainly found within $10^{\circ}$ of the geomagnetic equator. A small fraction of these waves observed at higher latitudes can be explained by the influence of instrumental noise. These results indicate, consistent with the subjective experience gained from the visual inspection of the entire data set, that the low-ellipticity criterion is able to identify equatorial noise with a reasonable success.

Equatorial noise has been shown to have the most intense magnetic field fluctuations among all the natural emissions in the given interval of frequencies and latitudes, being detected in approximately $80 \%$ of the cases where the magnetic power-spectral density exceeds $10^{-4} \mathrm{nT}^{2} \mathrm{~Hz}^{-1}$. Electric field fluctuations of equatorial noise are more intense compared to the average of all detected waves but their relative fraction decreases at very high intensities of more than
$10^{-2} \mathrm{mV}^{2} \mathrm{~m}^{-2} \mathrm{~Hz}^{-1}$, owing to the $95 \%$ domination of the broad band electrostatic noise.

The relatively high observed intensities and high occurrence ratios of equatorial noise indicate that these natural emissions can play a role in the dynamics of energetic ions in the internal magnetosphere. More research is needed to quantify their influence.

Acknowledgements. We sincerely thank Chris Harvey of CESR Toulouse, Michel Parrot of LPCE Orleans, Milan Maksimovic of the Meudon Observatory, and other colleagues from the STAFF team for construction, calibration and operations of the instrument and for fruitful discussions of the data. We gratefully acknowledge "space weather" discussions with François Lefeuvre of LPCE Orleans, who significantly influenced the principal direction of this research. Our thanks are due to the PI of the FGM instrument (A. Balogh) for the DC magnetic field data used for reference and coordinate transformations, and to the Hungarian Cluster Data Center which provides auxiliary data. ESA and CNES are thanked for their support for the STAFF experiment. This research was supported by the Czech Grant Agency grant No. 202/03/0832.

Topical Editor T. Pulkkinen thanks a referee for his help in evaluating this paper.

\section{References}

André, R., Lefeuvre, F., Simonet, F., and Inan, U. S.: A first approach to model the low-frequency wave activity in the plasmasphere, Ann. Geophys., 20, 981-996, 2002.

Balogh, A., Carr, C. M., Acuña, M. H., et al.: The Cluster Magnetic Field Investigation: overview of in-flight performance and initial results, Ann. Geophys., 19, 1207-1217, 2001.

Cornilleau-Wehrlin, N., Chauveau, P., Louis, S., et al.: The Cluster spatio-temporal analysis of field fluctuations (STAFF) experiment, Space Sci. Rev., 79, 107-136, 1997.

Cornilleau-Wehrlin, N., Chanteur, G., Perraut, S., et al.: First results obtained by the Cluster STAFF experiment, Ann. Geophys., 21, 437-456, 2003.

Gurnett, D. A.: Plasma wave interactions with energetic ions near the magnetic equator, J. Geophys. Res., 81, 2765-2770, 1976.

Horne, R. B., Glauert, S. A., and Thorne, R. M.: Resonant diffusion of radiation belt electrons by whistler-mode chorus, Geophys. Res. Lett., 30, 9, 1493, doi:10.1029/2003GL016963, 2003.

Horne, R. B. and Thorne, R. M.: Relativistic electron acceleration and precipitation during resonant interactions with whistler-mode chorus, Geophys. Res. Lett., 30, 10, 1527, doi:10.1029/2003GL016973, 2003.

Kasahara, Y., Kenmochi, H., and Kimura, I.: Propagation characteristics of the ELF emissions observed by the satellite Akebono in the magnetic equatorial plane, Radio Sci., 29, 751-767, 1994.

Laakso, H., Junginger, H., Roux, A., Schmidt, R., and de Villedary, C.: Magnetosonic waves above $f_{\mathrm{c}}(\mathrm{H}+)$ at geostationary orbit: GEOS 2 results, J. Geophys. Res., 95, 10 609-10 621, 1990.

Meredith, N. P., Cain, M., Horne, R. B., Thorne, R. M., Summers, D., and Anderson, R. R.: Evidence for chorus-driven electron acceleration to relativistic energies from a survey of geomagnetically disturbed periods, J. Geophys. Res., 108, A6, 1248, doi:10.1029/2002JA009764, 2003.

Perraut, S., Roux, A., Robert, P., Gendrin, R., Sauvaud, J.-A., Bosqued, J.-M., Kremser, G., and Korth, A.: A systematic study of ULF waves above $F_{\mathrm{H}^{+}}$from GEOS 1 and 2 measurements 
and their relationships with proton ring distributions, J. Geophys. Res., 87, 6219-6236, 1982.

Russell, C. T., Holzer, R. E., and Smith, E. J.: OGO 3 observations of ELF noise in the magnetosphere: 2 . The nature of the equatorial noise, J. Geophys. Res., 73, 755-768, 1970.

Samson, J. C.: Descriptions of the polarization states of vector processes: Applications to ULF magnetic fileds, Geophys. J. R. Astron. Soc., 34, 403-419, 1973.
Santolík, O., Pickett, J. S., Gurnett, D. A., Maksimovic, M., and Cornilleau-Wehrlin, N.: Spatiotemporal variability and propagation of equatorial noise observed by Cluster, J. Geophys. Res., 107, A12, 1495, doi:10.1029/2001JA009159, 2002.

Santolík, O., Parrot, M., and Lefeuvre, F.: Singular value decomposition methods for wave propagation analysis, Radio. Sci., 38, 1, 1010, doi:10.1029/2000RS002523, 2003.

Stix, T. H.: Waves in Plasmas, Am. Inst. of Phys., New York, 1992. 